\title{
Application of Artificial Neural Network in Green Building Project Cost Budget
}

\author{
Zhang Yueyue*, Chen Xiaohong \\ Railway Engineering College, ZhengZhou Railway Vocational\&Technical College, \\ Zhengzhou, Henan, China \\ *Corresponding Author.
}

\begin{abstract}
:
Construction cost budget is very important for enterprises, and its accuracy directly affects the profit rate of construction enterprises. An important factor affecting the accuracy of construction cost is the price fluctuation of building materials. In this paper, taking construction engineering and building materials as an example, fuzzy control and artificial neural network are used to predict the price of building materials. The results show that: artificial neural network can effectively predict the future price trend of building materials. Thus, the prediction method can provide the price trend of building materials for the project cost budget, and improve the accuracy of the cost budget.
\end{abstract}

Keywords: Construction Engineering, Cost Budget, Fuzzy Control, Artificial Neural Network.

\section{INTRODUCTION}

Building a well-off society in an all-round way is a major task of our country in the past decade [1-2]. Accordingly, China has also issued many documents for policy support, such as the national new urbanization plan and the 13th five year plan, which respectively put forward the goal of achieving $60 \%$ urbanization rate in seven years from 2014 to 2020 [3]. From 2016 to 2020, the rural population with the ability to live in the city should be promoted to settle down in the city and work [4-5]. Urbanization is the main potential to expand domestic demand in the historical process of building a well-off society in China. Urbanization can show the role of urban cluster mode, rich first and then rich, big cities drive small cities, gradually promote development, and realize real human urbanization. However, the most important material carrier in the process of urbanization is the construction industry [6]. Urban environment and housing prices are related to people's livelihood. Real estate enterprises can be said to act as the driving force of urbanization.

The real estate industry is developing rapidly with the pace of the times, and there is a real estate boom. In the process of rapid development, there are many problems, among which the estimation of project cost can not be ignored. Whether the estimation result is lagging or with large error, it may bring inestimable serious consequences to the investment decision-making, construction progress, construction quality of the project, and directly lead to the economic losses of the project participants. In order to ensure the smooth progress of the project and the economic benefit of the project investment, the cost estimation is the premise. 


\section{THE CONSTRUCTION OF PREDICTION MODEL}

\subsection{Selection of input neurons based on fuzzy logic}

1) Analysis of influencing factors of project cost

The total investment of construction project is large, the integrity is strong, the construction period is long, and there are many risk factors [7-10]. It is a specific process with start and end dates, which is composed of survey and design, procurement and construction, completion acceptance, handover and operation and other related activities. It is precisely because of these characteristics of construction projects, there are many factors that affect the project cost in the long process of project construction.

The general components of civil engineering are: foundation, column, beam, wall, floor panel, roof panel, stairs, doors and windows, etc. The cost of construction project is closely related to the quantity and price of these components, and the quantity of these components depends on the design parameters and structural parameters of the project. The price is related to the current market environment. This paper studies the movement and change law of the construction cost information, and regards the construction cost as an abstract open system, which is affected by the external environment and the internal attributes of the system. The external environment includes human factors, natural factors and market factors, while the internal attributes are the design parameters and characteristics of construction engineering, as shown in Figure 1.

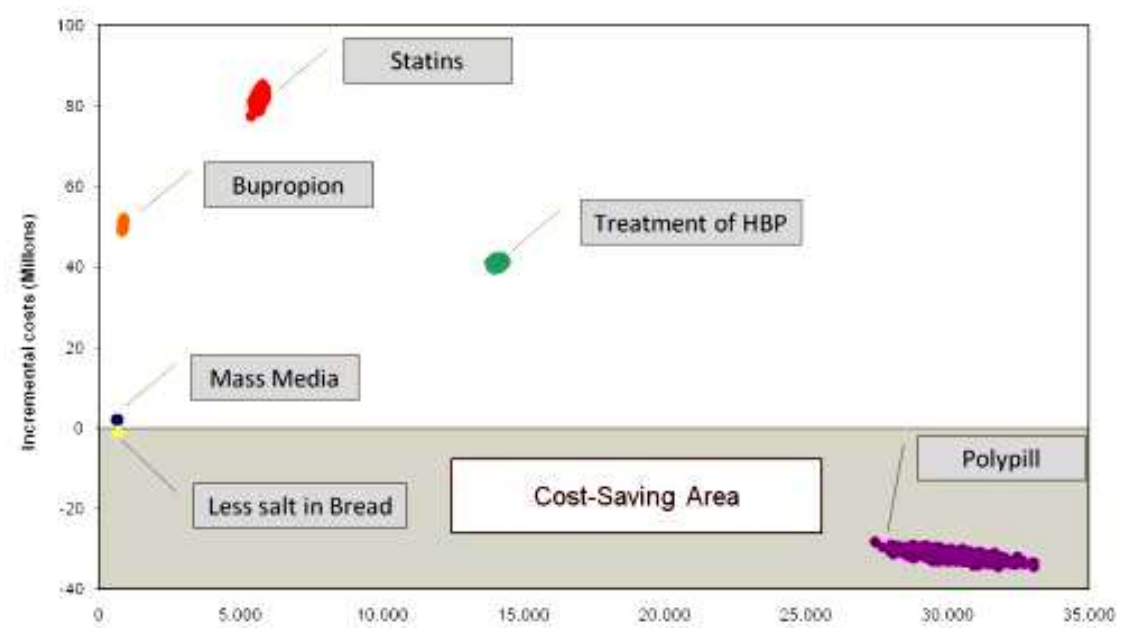

Fig 1: System diagram of influencing factors of construction cost

2) Construction of fuzzy logic model

The following principles should be followed when constructing the index system of construction cost prediction.

(1) Scientific principle. The establishment of construction project cost prediction index system should adopt the way of combining theory with practice, quantitative and qualitative analysis, so as to grasp the essence and connotation of things, and scientifically construct the construction project cost index system.

(2) The principle of comprehensiveness. The construction project cost prediction index should reflect the overall situation of a construction project. Therefore, each index should reflect one or more characteristics of the construction project and be combined. And can reflect the difference of each construction engineering characteristics.

(3) The principle of selectivity. The construction engineering entity is composed of many parts and sub projects, and its constituent elements have many kinds and characteristics. If all the characteristics are input 
as the variables of the prediction model, the structure will be complicated and have no practical significance. Therefore, in the process of building construction cost prediction index system, we should adopt the "two eight law" and select the index selectively and pertinently.

(4) The principle of independence. There are many characteristics of construction engineering, so there will inevitably be correlation or inclusion relationship between the corresponding characteristic indexes, which will affect the accuracy of the prediction results. Therefore, each construction cost prediction index should be as independent as possible to prevent mutual interference.

2.2 The cost prediction model based on Neural Network

1) The determination of network topology

The number of neurons on the hidden layer has a great influence on the prediction performance of neural network. Too few of them will reduce the ability of network analysis and clustering so that it can not accurately fit the nonlinear law, and the excessive number of neurons will appear over fitting phenomenon, which increases the calculation load and time of the network and reduces the operation efficiency. There are some rules for determining the number of nodes in the hidden layer: if the function to be fitted fluctuates violently and the connection weight is required, the number of nodes should be increased; if the precision is high, the number of nodes in the hidden layer should be increased; in addition, a little node can be added gradually or gradually banned in the process of sufficient addition at the beginning. These rules are often not universal and inaccurate. When scholars carry out research work on the basis of these rules, they often determine them according to empirical formula. The Kolmogorov theorem determines the number of nodes in the hidden layer according to the number of input layer nodes and the number of output layer nodes.

For the three-layer neural network, $n$ is the number of input layer nodes, $n=5$, the number of hidden layer nodes is $2 n+1=11$, and the number of output layer nodes $m$ is determined by the type of sample target data, $\mathrm{m}=1$. The model structure is shown in Figure 2 .

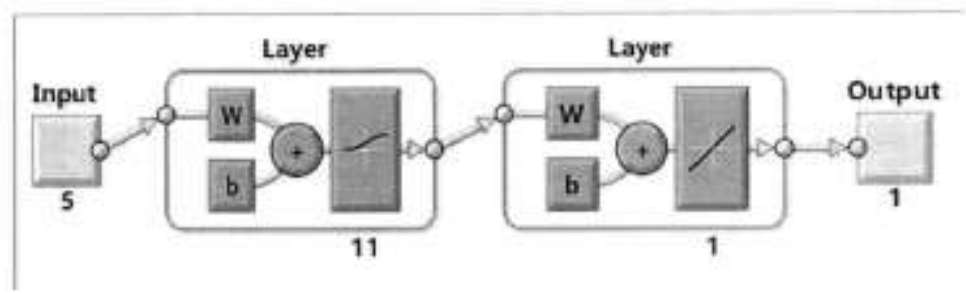

Fig 2: Network topology diagram

2) Network learning algorithm process

The steps of the standard learning algorithm of BP neural network are as follows

Step 1: network initialization

The topological structure of the network is determined, each connection weight is assigned a random number in the interval $(-1,1)$, the error function $\mathrm{e}$ is set, the calculation accuracy value $\varepsilon$ and the maximum learning times $\mathrm{M}$ are given.

Step 2: randomly select the k-th input sample and the corresponding expected output.

$$
\begin{array}{r}
x(k)=\left(x_{1}(k), x_{2}(k), \ldots, x_{n}(k)\right) \\
d_{o}(k)=\left(d_{1}(k), d_{2}(k), \ldots, d_{m}(k)\right)
\end{array}
$$

Step 3: Calculate the input and output of each neuron in the hidden layer. 


$$
\begin{array}{r}
h i_{h}(k)=\sum \omega_{i h} x_{i}(k)-b_{h}, h=1,2, \ldots, p \\
h o_{h}(k)=f\left(h i_{h}(k)\right), h=1,2, \ldots, p \\
y i_{h}(k)=\sum \omega_{h o} h o_{h}(k)-b_{o}, o=1,2, \ldots, m \\
y o_{o}(k)=f\left(y i_{o}(k)\right), o=1,2, \ldots, m
\end{array}
$$

\section{EMPIRICAL RESEARCH}

3.1 The realization of calculation

MATLAB language is convenient and efficient, and its rich function library and toolbox make developers save a lot of repeated programming. In this paper, the realization of mathematical modeling is completed by MATLAB.

MATLAB language is different from other high-level languages. It is called the fourth generation computer language, which liberates people from the tedious program code. Its rich function library saves developers a lot of repetitive programming work, and its biggest feature is simple and fast.

1) Easy to use, high programming efficiency

As a high-level language for scientific and engineering computing, it allows programming in mathematical form. Compared with other languages, it integrates editing, compiling, linking and execution. Input matlab command in the command line, including the statement of calling $m$ file. Each statement will be processed immediately to complete the whole process of compiling, connecting and running. If there is an error in the running, the platform will give detailed error information, so that it can be modified and executed until it is correct.

2) Good portability and openness

Matlab can be easily transplanted to the operating platform that can run $\mathrm{C}$ language, and there are many suitable working platforms. All the core files and toolbox of MATLAB are readable and writable source files, and they are open. Users can modify the source files and program to form a new matlab toolbox.

3) The sentence is concise and rich in meaning

The most basic component of MATLAB language is function statement. A function consists of function name, input variable and output variable. With the same function name, different numbers of input variables, including no input variables and different numbers of output variables, represent different functions and meanings, a bit like polymorphism in object-oriented. This not only makes the library function more functional, but also greatly reduces the disk space, making the file simple, short and efficient.

\subsection{Application of this model}

The simulation analysis of the construction cost prediction model based on fuzzy logic and neural network method is completed on the MATLAB operating platform. Matlab means matrix laboratory, which is developed from matrix operation. Its numerical calculation ability is second to none. It is a visual interactive programming platform, which overcomes the problems of $\mathrm{C}$ language, FORTRAN and other traditional non interactive languages, such as cumbersome procedures, and improves the work efficiency. Many toolkits are 
placed in the platform for users to call according to their needs. The interface is friendly and visual, the language is clear and concise, and the operation is convenient and fast.

Open matlab2012b platform operation: after calling the neural network toolbox in MATLAB to write the network, input the top 10 groups of sample data, repeatedly learn and train in the network, and constantly adjust and optimize the prediction performance of the neural network. The training convergence process is as follows. It can be seen from the training performance curve of the neural network (Fig. 3) that the network converges continuously in the training process, and the relative error shows a downward trend of fluctuation until it meets the accuracy requirements at 90 steps.

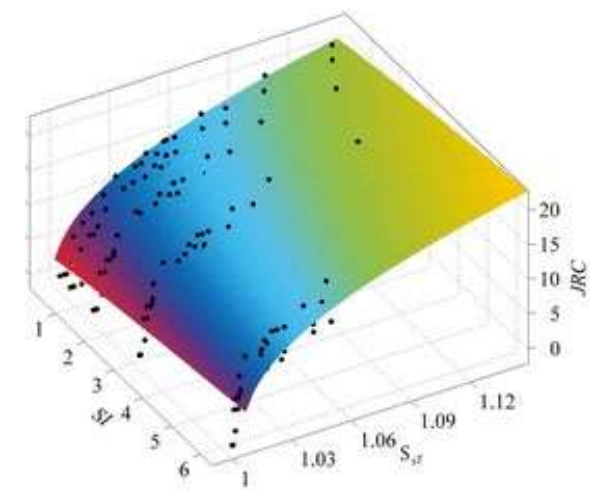

Fig 3: Training performance curve

The index data of the remaining two groups of sample data are input into the trained neural network for prediction, and the predicted value is inversely normalized by using the postmnmx function. Then the predicted result is compared with the actual value of the sample, and the relative error between the actual value and the predicted value is calculated.

TABLE I. Error analysis table

\begin{tabular}{|c|c|c|}
\hline ESTIMATE & ACTUAL VALUE & RELATIVE ERROR /\% \\
\hline 5028420 & 5194560 & 3.2 \\
\hline 14962470 & 16152640 & 7.37 \\
\hline
\end{tabular}

The results are as follows: 1 . The error is within $\pm 10 \%$, which meets the highest standard of investment estimation error rate of engineering feasibility study analysis. The model can be applied to cost prediction.

3.3 Traditional model application

The difference between the pure BP neural network model without fuzzy logic analysis and the model in this paper is that the input vector is not screened by fuzzy logic analysis. The design parameters in the design stage, i.e. the engineering characteristics, are selected as the index system to analyze the influence of the cost, which are: building area, building storey height, standard floor area, building storey number, structure type, plane shape, seismic grade, foundation type and foundation buried depth.

It means different topological structures. Similarly, according to Kolmogorov theorem, for a three-layer neural network, $n$ is the number of input layer nodes, $n=9$, the number of hidden layer nodes is $2 n+1=19$, and the number of output layer nodes is determined by the type of target data of the sample, $m=1$. The model structure is shown in Figure 4. 


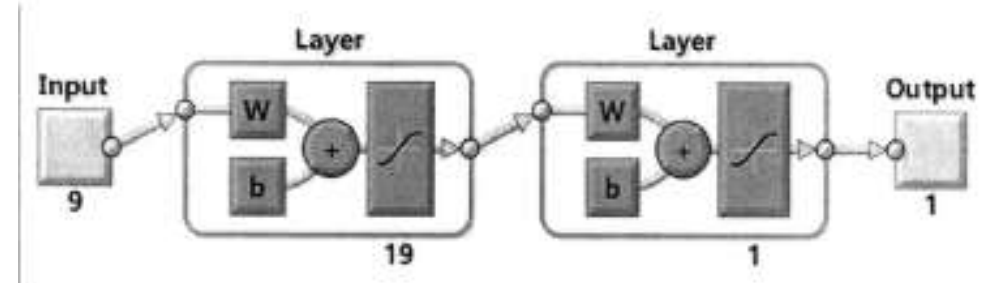

Fig 4: Network topology diagram

Open MATLAB2012b platform for operation, call the neural network toolbox in MATLAB to compile the network, input the first 10 groups of sample data, study and train repeatedly in the network, and constantly adjust and optimize the prediction performance of neural network. The convergence process of training is as follows. From the training performance graph of neural network (Figure 5), it can be seen that the relative error of the network keeps converging during the training process, and the accuracy requirement is met until 1000 steps.

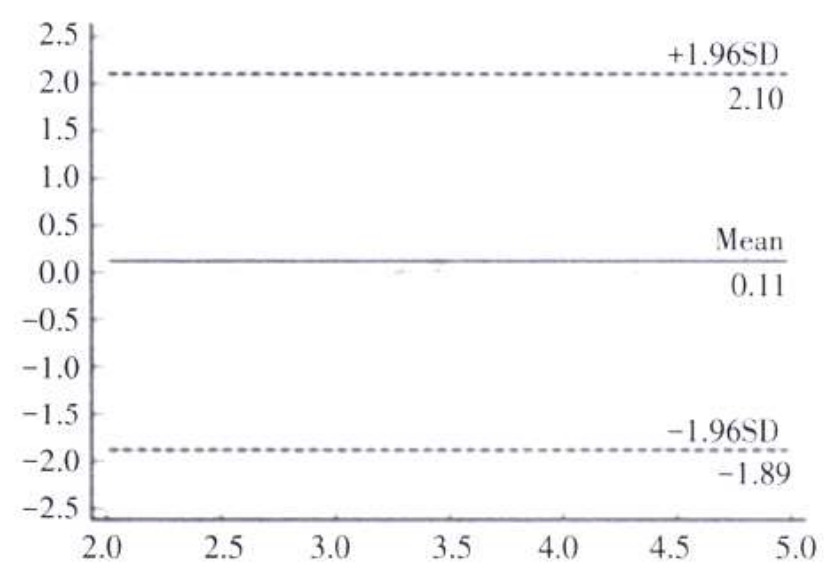

Fig 5: Training performance graph

The index data of the remaining two groups of sample data are input into the trained neural network for prediction, and the predicted value is inversely normalized by using the postmnmx function. Then the predicted result is compared with the actual value of the sample, and the relative error between the actual value and the predicted value is calculated.

TABLE II. Error analysis table

\begin{tabular}{|c|c|c|}
\hline ESTIMATE & ACTUAL VALUE & RELATIVE ERROR /\% \\
\hline 5572724 & 5194560 & 7.28 \\
\hline 17490156 & 15752640 & 11.03 \\
\hline
\end{tabular}

\section{CONCLUSION}

In this paper, combined with fuzzy logic analysis method and neural network analysis method, the application of construction cost prediction model is studied. There are many factors influencing the construction cost, which also have the characteristics of dynamic, hierarchical, differential, large amount and compatibility. The selection of the index system of the project cost is a problem under the fuzzy environment 
with uncertainty. The weights obtained only by the relevant experts according to the experience are not scientific, and the hesitation in the decision-making is not considered. Therefore, the application of fuzzy logic analysis method to the selection of the index system is more scientific and reasonable. The same sample data is used in the fuzzy logic + neural network prediction model, multiple linear regression prediction model and traditional BP neural network prediction model respectively to obtain the predicted value, calculate the relative error between the predicted value and the actual value, and then compare and analyze its accuracy. The results show that: the prediction accuracy ratio of multiple linear regression model is the worst; the generalization ability of neural network model is poor, and the performance is unstable; the prediction accuracy of paste neural network + neural network method is the highest, and it overcomes the shortcomings of low precision of multiple linear regression model and poor generalization ability of neural network model.

\section{REFERENCES}

[1] Tan Zhongsheng, Yang Xiaolin, Wang Mengshu. Analysis of the Influence of Blasting on the Existing Tunnel in Double Track Tunnel Construction. Journal of Rock Mechanics and Engineering, 2003, 7:66-69

[2] Yang Junsheng, Liu Baochen. Ground Movement and Deformation Caused by Construction of Extrusion Shield Tunnel. Geotechnical Mechanics, 1998 (03): 10-13

[3] Wu Bo, Gao Bo. Study on Surface Settlement of Urban Subway Tunnel Construction Under Complex Conditions. China Railway Science, 2006, 27 (006): 129-131

[4] Han Xuan, Li Ning. Applicability Analysis of Peck Formula in Ground Deformation Prediction of Tunnel Construction in China. Geotechnical Mechanics, 2007, 28 (01):121-127

[5] Li Zhucai, Li Chen, Xue Yiguo. Study on the Key Technology of Comprehensive Prediction and Early Warning of Tunnel Construction Geological Hazards in High Risk Karst Area. Journal of Rock Mechanics and Engineering, 2008, 27 (007): 1297-1307

[6] Yang Xiaohua, Yu Yonghua. Application of Cement Water Glass Double Liquid Grouting in Loess Tunnel Construction. Chinese Highway Journal, 2004, 9:107-112

[7] Huang Hongwei, Zeng Ming, Chen Liang. Development of Risk Management Software for Shield Tunnel Construction (trm1.0) Based on Risk Database. Journal of Underground Space and Engineering, 2006, 2 (1): $36-41$

[8] He Yu, Li Fuming, Xiao Yang. Study on Advance Prediction Technology of Tunnel Construction Crossing Syncline. Journal of Underground Space and Engineering, 2020, V.16; No.126 (s1): 492-497

[9] Li Yunpeng, Wang Zhiyin, Han Changling. Simulation Study on Construction Process of Small Spacing Tunnel with Different Surrounding Rock Types. Geotechnical Mechanics, 2006 (01): 11-16

[10] Wu Bo, Liu Weining, Gao Bo. Analysis of Temporal and Spatial Effects of Construction Behavior of Urban Shallow Tunnel. Chinese Journal of Geotechnical Engineering, 2004, 026 (003): 340-343 